\title{
Production of High Power Femtosecond Terahertz Radiation
}

\author{
George R. Neil ${ }^{(1) *}$, G. L. Carr ${ }^{(2)}$,Joseph F. Gubeli III ${ }^{(1)}$, K. Jordan $^{(1)}$, Michael \\ C. Martin ${ }^{(3)}$, Wayne R. McKinney ${ }^{(3)}$, Michelle Shinn ${ }^{(1)}$, Masahiko Tani ${ }^{(4)}$, and \\ G. P. Williams ${ }^{(1)}, X .-C$. Zhang ${ }^{(5)}$ \\ ${ }^{(1)}$ Thomas Jefferson National Accelerator Facility, 12000 Jefferson Avenue, Newport News, VA 23606 USA \\ ${ }^{(2)}$ National Synchrotron Light Source, Brookhaven National Laboratory, Upton, NY 11973 \\ ${ }^{(3)}$ Advanced Light Source, Lawrence Berkeley National Laboratory, Berkeley, CA 94720 \\ ${ }^{(4)}$ Research Center for Superconductor Photonics, Osaka University, 2-1 Yamadaoko, Suita, Osaka 565-0871, Japan \\ ${ }^{(5)}$ Physics Department, Rensselaer Polytechnic Institute, 110 8th St., Troy, NY 12180-3590
}

\begin{abstract}
The terahertz $(\mathrm{THz})$ region of the electromagnetic spectrum is attracting interest for a broad range of applications ranging from diagnosing electron beams to biological imaging. Most sources of short pulse $\mathrm{THz}$ radiation utilize excitation of biased semiconductors or electro-optic crystals by high peak power lasers. For example, this was done by using an undoped InAs wafer irradiated by a femtosecond free-electron laser (FEL) at the Thomas Jefferson National Accelerator Facility. Microwatt levels of THz radiation were detected when excited with FEL pulses at $1.06 \mu \mathrm{m}$ wavelength and $10 \mathrm{~W}$ average power. Recently substantially higher powers of femtosecond THz pulses produced by synchrotron emission were extracted from the electron beamline. Calculations and measurements confirm the production of coherent broadband THz radiation from relativistic electrons with an average power of nearly 20 watts, a world record in this wavelength range by a factor of 10,000. We describe the source, presenting theoretical calculations and their experimental verification. Potential applications of this exciting new source include driving new non-linear phenomena, performing pump-probe studies of dynamical properties of novel materials, and studying molecular vibrations and rotations, low frequency protein motions, phonons, superconductor bandgaps, electronic scattering, collective electronic excitations (e.g., charge density waves), and spintronics.
\end{abstract}

PACS codes: 41.60.Cr, 52.75.Ms

Keywords: Linac, $\mathrm{THz}$, recirculation

\footnotetext{
* N eil, George R., Jefferson Lab, 12000 Jefferson A venue, Mail Stop 6A, phone: 757-269-7443, fax: 757-269-5519
} 


\section{Introduction}

The THz region, $\left(1 \mathrm{THz}=33 \mathrm{~cm}^{-1}\right.$ or $\left.4 \mathrm{meV}\right)$, lies in the far infrared spectral range where conventional thermal sources are very weak. For example, a $2000 \mathrm{~K}$ blackbody source typically provides less than 1 microwatt $/ \mathrm{cm}^{-1}$. While narrow band sources have been available using FEL technology[1], a significant advancement in broadband $\mathrm{THz}$ sources has occurred over the past decade with the advent of coherent $\mathrm{THz}$ radiation emission from photocarriers in a biased semiconductor or from optical rectification in an electro-optic crystal[2]. An energy per pulse of about $1 \mu \mathrm{J}$ has been achieved, implying MW peak powers, but at repetition rates of $1 \mathrm{kHz}$ such that average power levels are only $1 \mathrm{~mW}$ [3]. The JLab FEL was used to produce such radiation from a semiconductor while operating in the third harmonic at 1 micron[4].

This work describes a different process for producing coherent $\mathrm{THz}$ radiation from accelerated electrons. This process also begins with pulsed laser excitation in GaAs, but uses photoemission to produce bunches of electrons. Using the energy recovery linac (ERL) at the Jefferson Lab Free Electron Laser[5], electron bunches are brought to $40 \mathrm{MeV}$ in a linac and then transversely accelerated by a magnetic field to produce the $\mathrm{THz}$ emission as synchrotron radiation. This accelerator produces high average current (up to $5 \mathrm{~mA}$ ) by operating at a very high repetition rate (up to $75 \mathrm{MHz}$ ) using superconducting RF cavities and recovering the energy of the spent electrons. Electron bunches as short as $\sim 500 \mathrm{fs}$ are produced by the technique of energy modulation followed by compression in the dispersive region of a magnetic chicane.

Like the $\mathrm{THz}$ emitter described above, the electrons experience a common acceleration. If the electron bunch dimensions are small (in particular, the bunch length is less than the wavelength of observation), one obtains a multiparticle coherent enhancement $[6,7]$ of the form $\mathrm{N}[1-f(\omega)]+\mathrm{N}^{2}$, where $\mathrm{N}$ is the number of particles in a bunch and $f(\omega)$ is the Fourier transform of the normalized longitudinal particle distribution within the bunch. The power produced is given by Larmor's formula[8] which in CGS units takes the form:

$$
\text { Power }=\frac{2 e^{2} a^{2}}{3 c^{3}} \gamma^{4}
$$

where e is the charge, a is the acceleration, $\mathrm{c}$ the speed of light and $\gamma$ is the ratio of the mass of the electron to its rest mass. For a conventional Auston switch $\mathrm{THz}$ emitter based on a laser pulse striking GaAs, the voltage across the gap might produce an acceleration of the freed charges on the order of $10^{18}$ $\mathrm{cm} / \mathrm{sec}^{2}$. A similar acceleration results from a $1 \mathrm{~m}$ radius magnetic bend but in our case $\gamma$ is 75 , yielding a considerable enhancement in radiated power.

Such coherent synchrotron radiation has been observed from electrons accelerated in linacs[9-12], and more recently it has been discussed and observed from electron bunches in storage rings[13-17], but not at $\mathrm{THz}$ frequencies or in a form stable enough for use as a light source although there are several development efforts in place in this area[17, 18].

\section{Calculations And Results}

Details of the theory have been presented elsewhere[19]; in Fig. 1 we present calculations and measurements of the power emitted by a $500 \mathrm{fsec}$ fwhm electron bunch. We assumed the electron bunches had an energy of $40 \mathrm{MeV}$, a charge of $100 \mathrm{pC}$, and that they passed through a $1 \mathrm{~m}$ radius bend at a $37.4 \mathrm{MHz}$ repetition rate.

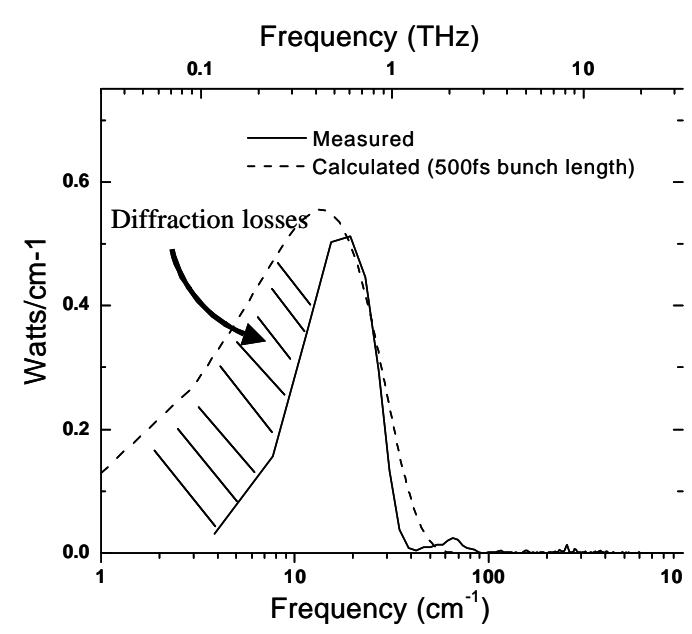

Figure 1. Calculated and measured spectral power. The measured spectrum suffers from diffraction losses at the long wavelength end. 
The spectral content of the emitted $\mathrm{THz}$ light was analyzed using a Nicolet 670 rapid-scan Michelson interferometer and detected using a $4.2 \mathrm{~K}$ Infrared Laboratories bolometer. Our collection angle was 60 $\times 60$ milliradians and the extraction window was quartz. We were able to determine the absolute power in two ways, one using a calibrated pyroelectric detector, and one by comparing our spectra with that from a $1300 \mathrm{~K}$ thermal source. The data has been scaled on the basis of these absolute power measurements. The spectral onset of the superradiant enhancement of the $\mathrm{THz}$ light is clearly seen on the high frequency side. The onset shape is also seen to match closely the theoretical predictions. Note that there is a severe discrepancy on the lower frequency side due to diffraction effects from the small aperture at long wavelengths.

We note that we were able to observe considerable changes in spectral weight depending on operating conditions, and were able to enhance certain spectral regions via the machine parameters. Thus the electron bunch distributions were not purely Gaussian but contained higher order components. Since we measured the intensity, not field, of the emitted light, we were unable to determine uniquely the electron density distributions, but this might be possible in future experiments using coherent detection. This can in principle be a powerful technique for the analysis of linac performance. For example noise spectral measurements (Figure 2) can be utilized to uncover sources of beam fluctuations.

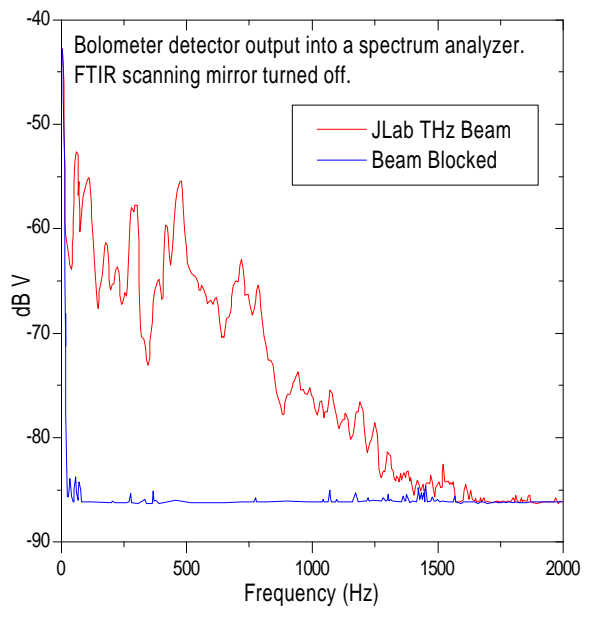

Figure 2. A measurement of noise on the $\mathrm{THz}$ signal was used to identify fluctuations in the drive laser power on the cathode.
Finally we measured the polarization of the emitted $\mathrm{THz}$ light. The intensity ratio for the horizontal to vertical polarization components is 3 for synchrotron radiation in the long-wavelength limit. This assumes full collection of the emitted radiation. We note that the dominant intensity is near $30 \mathrm{~cm}^{-1}$, which has a natural opening angle of 86 millirad. Since the emission pattern is "clipped" by the $60 \mathrm{mrad}$ collection optics, the calculated ratio is expected to be higher, approaching a value of 6 . Using a wire-grid polarizer placed between the Michelson interferometer and the detector, we measured a ratio of 5 (see Figure 3).

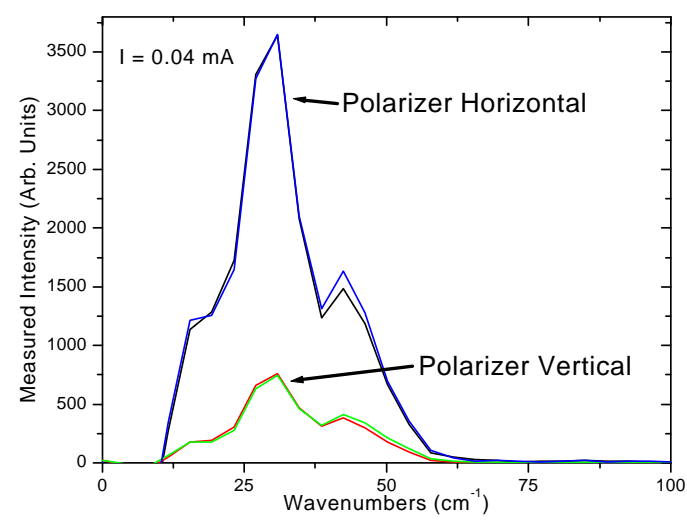

Figure 3. Spectral characteristics illustrating the structure that can result from electron density modulation at higher orders than the bunch length. The measured polarization ratio is in agreement with calculations.

The intensity of the radiation can be gauged from Figure 4, which is a real time image taken in $\mathrm{THz}$ radiation on a pyroelectric camera. Although detailed $\mathrm{THz}$ images have previously been obtained by a number of research groups, it typically took minutes to hours to obtain an image. The present source should permit real time imaging over large areas and/or determination of spectral absorption in a broad wavelength band simultaneously. 


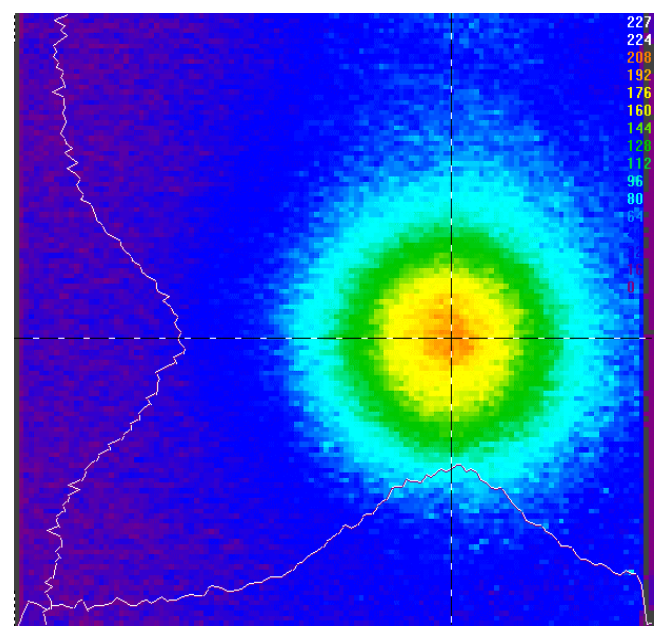

Figure 4. A real time image of the THz emission taken using a pyroelectric camera illustrates the available power.

\section{Conclusion}

We are now installing an upgrade to the facility at Jefferson Lab (see Neil, et al., in a separate talk at this conference) in which we will considerably improve the $\mathrm{THz}$ extraction aperture. $\mathrm{THz}$ radiation will be outcoupled through a port and delivered to an experimental facility for use independent of the FEL.

\section{Acknowledgements}

This work was supported primarily by the U.S. Dept. of Energy under contracts DE-AC0298CH10886 (Brookhaven National Laboratory), DEAC03-76SF00098 (Lawrence Berkeley National Laboratory) and DE-AC05-84-ER40150 (Thomas Jefferson National Accelerator Facility). The JLab FEL is supported by the Office of Naval Research, the Air Force Research Laboratory, the Commonwealth of Virginia and the Laser Processing Consortium. We are indebted to our colleagues at each institution for critical support.

\section{References}

[1] S. Winnerl, et al., Applied Physics Letters 77, 1259-1261 (2000).

[2] B. Ferguson and X.-C. Zhang "Materials for Terahertz Science and Technology," in Nature Materials, Sept. 1, 2002. (in press)

[3] D. You, R. R. Jones, P. H. Bucksbaum, and D. R. Dykaar, Optics Letters 18, 290292 (1993).

[4] Masahiko Tani, et al., Ultrafast Electronics and Optoelectronics Conference (UEO 2001), Lake Tahoe, NV, January 10-12, 2001.

[5] G.R. Neil, et al., Physical Review Letters 84, 662 (2000).

[6] S. Nodvick, D. S. Saxon, Phys. Rev. 96, 180-184 (1954).

[7] C.J. Hirschmugl, M. Sagurton, and G. P. Williams, Phys. Rev. A44, 1316-1320 (1991).

[8] J. D. Jackson, Classical Electrodynamics, Wiley, New York (1975).

[9] T. Nakazato, et al., Phys. Rev. Lett. 63, 1245-1248 (1989).

[10] U. Happek, E. B. Blum, and A. J. Sievers, Phys. Rev. Lett. 67, 2962-2965 (1991).

[11] D. X. Wang, G. A. Krafft, and C. K. Sinclair, Phys. Rev. E57, 2283-2286 (1998).

[12] Hung-chi Lihn, P.Kung, C. Settakorn, and H. Wiedemann, Phys. Rev. Letts. 76, 4163-4166 (1996).

[13] H. Tamada, H. Tsutsui, K. Shimoda, and K. Mima, Nuclear Instr. \& Meth. A331, 566 (1993).

[14] A. Andersson, M. S. Johnson, and B. Nelander, Optical Engineering, 39, 30993105 (2000)

[15] U. Arp, et al., Phys. Rev. Special Topics, Accelerators and Beams 4, 54401-154401-9 (2001)

[16] G. L. Carr, et al., Nucl. Instr. \& Meth. A463, 387-392 (2001).

[17] M. Abu-Bakr, et al., Phys Rev. Letts. 88, 254801 (2002).

[18] Giovenale, E., et al. Nucl. Inst. and Meth A437, 128-133 (1999).

[19] G. P. Williams, Rev. Sci. Instr. 73, 1461 (2002). 